Chapter 6

\title{
Pathways of Intrinsic Apoptosis in Neuroblastoma: Targets for Therapeutics and New Drug Development
}

\author{
Fieke Lamers and Aru Narendran \\ Additional information is available at the end of the chapter \\ http://dx.doi.org/10.5772/55817
}

\section{Introduction}

Neuroblastoma (NB) is one of the most difficult to treat malignancies of early childhood that originates from the sympathetic nervous system and ranks high among the diseases with unacceptable fatality rates in paediatrics. Currently, children with high risk NB are treated with intensive multi-modal therapeutic regimens, but often endure disease recurrence that is refractory to further treatment. Hence, research strategies are urgently needed to discover novel therapeutic targets to advance the timely development of innovative treatment approaches for these children.

In general, growth and survival of tumors are thought to be defined largely by deregulated genetic processes such as cell cycle checkpoints, DNA damage repair mechanisms, oncogenes and tumor suppressor genes, resulting in enhanced and unregulated malignant cellular proliferation. These findings have contributed significantly to the development of various chemotherapeutic agents and current treatment protocols. In addition, recent studies have provided evidence for enhanced tumor survival as a consequence of the breakdown of the cell death mechanisms that otherwise safeguard the integrity of normal tissue homeostasis while evading over-proliferation.

Reports from several laboratories have shown that NB cells carry defective or silenced proapoptotic factors, such as caspases (cysteinyl aspartate-specific proteases; CASP) and have enhanced expression and activity of a range of pro-survival factors [1]. These observations led to the reasoning that better understanding of the apoptotic mechanisms that sustain the survival of NB cells could aid in the development of novel therapeutic approaches. The potential to target and modulate the life or death signals in cancer cells carries immense therapeutic potential and therefore research continues to focus on the understanding of the 
apoptosis process that intersects the growth and survival pathways of NB. It is hoped that this information will facilitate effective therapeutic drug discoveries.

\section{Apoptosis}

Under normal circumstances, cell death processes are characterized by distinct morphological changes and are classified as necrotic, apoptotic, autophagic or those coupled with mitotic catastrophe. Among these, apoptosis relates to programmed cell death that occurs in response to distinct signals such as hypoxia, excessive oncogene activation or chemotherapeutic agents. The mechanistic basis for this process involves the concerted activity of caspases, which inactivate or activate target substrates in a cascade of enzymatic activities. This sequence of activities is broadly grouped as the "extrinsic" and the "intrinsic" apoptotic pathway. The extrinsic pathway involves the engagement of cell surface "death receptors", activated by extra-cellular signals, which induce apoptosis by directly activating the caspase cascade. The "intrinsic" pathway, also known as the mitochondrial apoptotic pathway, is activated from within the cell in response to signals of cellular stress. This may occur as a result of deprivation of cell survival factors, DNA damage and increased levels of abnormally folded cellular proteins and reactive oxygen species [2], Figure 1. This process, in conjunction with the proapoptotic BCL2 family mediated pore formation, leads to the release of mitochondrial mediators such as DIABLO (SMAC) and CYCS (cytochrome c) [2]. Once released, CYCS complexes with APAF1 to mediate dATP/ATP dependent activation of APAF1 and proCASP9, leading to subsequent caspase activation, cell death and more release of DIABLO. However, this process also lends to the liberation of inhibitor-of-apoptosis protein (IAP) mediated inhibition of the pro-caspases [3-5]. Currently, however, it appears that in some cell types, alternate pathways can contribute to the cellular apoptotic activity.

\section{BCL2 family of apoptosis regulators}

By virtue of their ability to localize to mitochondrial membranes, the BCL2 family of proteins play a pivotal role in the regulation of mitochondrial apoptotic pathways [6]. They share at least one of four homologous regions known as BCL homology (BH) domains (BH1-BH4), which enable the formation of homo- and heterotypic dimers among these molecules. All antiapoptotic effectors and members and some pro-apoptotic members, such as BAX and BAK1, share sequence homology of three or more of such domains, whereas the BH3-only proteins show sequence homology only within the BH3 domain [6, 7]. Such interactions are thought to form the mechanistic basis for the activity of BCL2 proteins. These proteins can be divided into anti-apoptotic members, including BCL2, BCL2L1 (BCL-XL), MCL1 and BCL2L2 (BCL-W), and pro-apoptotic members. The pro-apoptotic members can be divided into three groups: 1 . proteins with multi-domain members: BAX and BAK1, which form pores in the mitochondrial membrane through which CYCS and DIABLO can be released, 2. the group of BH3-only members including proteins that inhibit anti-apoptotic members by binding directly, such as 
PMAIP1 (NOXA), BAD and BIK and 3. the collection of pro-apoptotic BH3-only proteins that can either inhibit the anti-apoptotic members or induce BAX/BAK pore formation directly. This last group consists of BID, BCL2L11 (BIM) and BBC3 (PUMA) [6-8].

In cancer cells, the BCL2 family of proteins contribute to enhanced cell survival and expansion by blocking physiologically relevant cell death processes. Up-regulated BCL2 proteins also play a key role in the generation of resistance to chemotherapeutic drugs and radiotherapy by interfering with tumor cell death induced by cytotoxic agents [9]. In addition, they also offer protection against cell death pathways that are activated during conditions such as cytokine withdrawal. An altered expression of BCL2 proteins has been found in many cancers, including NB [10, 11]. Furthermore, transfection mediated over-expression of BCL2 or BCL2L1 in NB cells has been shown to generate a phenotype with acquired resistance to therapeutic agents [12]. Overall, current experimental evidence suggest that BCL2 expression critically regulates apoptosis and plays an important role in the tumorigenesis and survival of NB [13].

B-cell lymphoma-extra-large (BCL2L1, BCL-XL) is a mitochondrial membrane protein and a member of the BCL2 family. BCL2L1 has been shown to exhibit its anti-apoptotic properties by regulating mitochondrial homeostasis. Over-expression of BCL2L1 confers a multidrug resistance phenotype and protects tumor cells from chemotherapy induced differentiation and apoptosis. A recent study has shown that, in NB cells, repression of BCL2L1 by the proteasome inhibitor bortezomib resulted in the activation of pro-apoptotic PMAIP1, thereby triggering cell death [14]. Additional studies have shown that targeted inhibition of BCL2L1 in combination with 4-HPR (a synthetic retinoid) can work synergistically to significantly increase differentiation and apoptosis in BCL2L1 bountiful NB cells [15, 16]. These data provide rationale for targeting regulatory pathways of BCL2 proteins in therapeutic approaches for NB patients.

\section{Inhibitor of Apoptosis Proteins (IAPs)}

The inhibitor of apoptosis proteins are a group of conserved molecules that are frequently over-expressed in tumors that confer survival properties and chemotherapy resistance [17, 18]. Structurally, these proteins are characterized by one to three baculoviral IAP repeats (BIR) domains, which carry characteristic caspase inhibitory activity. The known members of the human IAP family include, NAIP (BIRC1), c-IAP1 (BIRC2), c-IAP2 (BIRC3), XIAP (BIRC4), survivin (BIRC5), Apollon/Bruce (BIRC6) ML-IAP (BIRC7 or livin) and ILP-2 (BIRC8) [19]. IAPs appear to control both extrinsic and intrinsic apoptotic pathways. By virtue of their ubiquitin ligase activity, BIRC2 and BIRC3 regulate the extrinsic apoptotic pathway [20]. As for the effects on the intrinsic pathway, XIAP inhibits CASP3, CASP7 and CASP9 by direct binding. However, this activity can be diminished by DIABLO binding to XIAP through its N-terminal IAP-binding motif (IBM) [21]. Furthermore, the activity of DIABLO can be blocked by BIRC5 which can also bind and stabilize XIAP $[22,23]$.

BIRC5 (MW 16.5-kDa) is an IAP member protein found in dividing cells that carries at least one BIR domain and normally exists as a homodimer [24]. The expression of BIRC5 has been 
demonstrated in many diverse tumor types, including neuroblastoma and appears to correlate with poor prognosis [25]. Many potential mechanisms have been postulated for the regulation of cellular expression of BIRC5 in cancer cells, including its transcriptional repression by wildtype p53, gene amplification, hypomethylation, increased promoter activity, and loss of p53 function [26, 27]. BIRC5 appears to have multiple functions in the growth and survival of tumor cells [28]. Although not shown in all experimental systems, some studies have indicated a role for BIRC5 in the regulation of cellular caspase activity. For example, a report by Tamm and colleagues showed that BIRC5 can be co-immunoprecipitated with CASP3, CASP7, and CASP9 and it suppresses apoptosis following over-expression of these caspases [29]. In staurosporine (STS)-induced apoptosis in NB model, BIRC5 has been shown to exert its phase specific antiapoptotic effect by inhibiting CASP9 activity [30]. Recently, using affymetrix mRNA expression analysis, a strong up-regulation of BIRC5 in NB cells compared to normal and fetal adrenal tissues and adult tumor specimens has been demonstrated [31]. Increased BIRC5 levels were also found to be associated with poorer prognosis, independent of chromosome 17q gain. Furthermore, antisense mediated silencing of BIRC5 in ten NB cell lines showed significantly increased apoptotic cell death defined by PARP cleavage and loss of cell viability.

In addition to its influence on programmed cell death, BIRC5 has also been shown to be a component of the chromosome passage protein complex (CPC), which is needed for chromosome alignment and segregation during mitosis and cytokinesis. The remaining constituents of CPC include AURKB (Aurora-B kinase), CDCA8 (Borealin), and INCENP [32]. Based on localization findings, it has been postulated that nuclear BIRC5 is involved in the control cell division, whereas cytoplasmic/mitochondrial BIRC5 is cytoprotective [33]. Constitutive expression of BIRC5 has also been demonstrated in a number of neuroblastoma cell lines [27]. BIRC5 knockdown in SK-N-BE2 and SH-SY-5Y NB cells caused an increase in expression of pro-apoptotic BAX and a decrease in anti-apoptotic BCL2 expression. A recent study by Miller and colleagues examined the relationship between CASP8 and BIRC5 levels and outcomes in neuroblastoma patients [34]. In this investigation, increased BIRC5 was found to be associated with poor overall survival and an increased BIRC5 to CASP8 ratio was associated with unfavorable histology and high risk stratification, indicating a combined influence of these two apoptosis associated factors in the clinical consequences of NB. Moreover, additional studies have shown that CASP8 is often hypermethylated in neuroblastoma tumors resulting in an inactive extrinsic apoptotic pathway [35-37].

BIRC7 is a member of the IAP family that has been found to play a notable role in apoptosis [38]. The expression of BIRC7 has been demonstrated in NB tumor specimens and cell lines [39]. Although the expression of BIRC7 by itself does not appear to be a prognostic marker, patients with increased BIRC7 expression and MYCN amplification had significantly poorer survival compared to those lacking both or either one of these markers. This suggests that NB patients with increased BIRC7 and MYCN may constitute a worse prognosis subset within the MYCN amplified group. Subsequently it has been shown that in cells that have increased MYCN and BIRC7, the suppression of MYCN leads to loss of BIRC7 [40]. An opposite effect was also seen when NB cells with low MYCN were induced to up-regulate MYCN, which led to increased BIRC7 levels. Furthermore, these studies also detected a consensus MYCN binding domain within the 5' proximal sequence of the putative BIRC7 promoter, indicating 
that MYCN is involved in the expression of BIRC7 and that BIRC7 may offset the effects of MYCN. Normally, NB cells with MYCN amplification show increased proliferation and paradoxically, increased sensitivity to apoptosis by chemotherapeutic agents [41]. Data provided by Dasgupta and colleagues suggest that MYCN may act as a transcriptional activator of BIRC7 expression and in cells co-expressing these genes, the anti-apoptotic effect of BIRC7 may counteract the apoptotic effects of MYCN amplification, thus enabling tolerance to cytotoxic agents and enhancing tumor growth and survival properties [42].

BIRC6 (also known as BIR-containing protein 6, Bruce or Apollon) is a giant $528 \mathrm{kDa}$ highly conserved protein that has been implicated as a modulator of the intrinsic apoptotic pathway promoting cell survival. The apoptosis inhibitory functions of BIRC6 is mediated by its ability to bind to caspases through its BIR domain. In humans, BIRC6 has been shown to be involved in the generation of chemotherapy resistance in cancer cells [43]. In vitro studies have shown its ability to ubiquitylate DIABLO and consequently cause hindrance to apoptosis caused by DIABLO [44].Inaddition, BIRC6 also binds to pro-CASP9 and inhibitsits cleavage and activation [45]. The expression of BIRC6 in cancer has been investigated in a number of recent studies. For example, an up-regulation of BIRC6 has been found in gliomas that are resistant to treatment [43] and in pediatric ALL [46], where its over-expression appears to be associated with poor overall and disease free survivals. Gene copy number gains and increased expression of BIRC6 in primary NB specimens have been shown the silencing of BIRC6 leads to cell death in the NB cell line SKNSH [47]. Importantly, these studies have demonstrated that in neuroblastoma cells, BIRC6 binds to DIABLO and that DIABLO levels increase upon silencing of BIRC6, indicating a mechanism for the degradation of cytoplasmic DIABLO by BIRC6.

\section{Targeted drug development}

Experimental evidence regarding the role of the BCL2 family of proteins in the intrinsic apoptotic pathway of NB led to the evaluation of agents that are BH3 mimetics. These drugs compete with $\mathrm{BH} 3$ domains for interaction with the apoptosis inhibitors and prevent the inhibitors from sequestering the pro-apoptotic members [Reviewed in 48]. Prominent among these are ABT-737 and it's orally bioavailable analog, ABT-263. These small molecule inhibitors bind to BCL2, BCL2L1 and BCL2L2 with high affinity and induce apoptosis as single agents or in combination with chemotherapeutic agents based on the priming status of the inhibitors [49]. Studies by Klymenko et al showed that ABT-737 sensitizes NB cells to clinically relevant cytotoxic agents under normoxic conditions and maintains its activity under hypoxia, when tumor cells show resistance to these agents [49]. Using a BH3 profiling approach with mitochondria isolated from NB cells, Goldsmith and colleagues have demonstrated that such profiles can accurately predict whole cell sensitivity to small molecule BCL2 family antagonists and may be useful in predicting response to agents, thereby targeting chemoresistance in NB [50]. Several studies have evaluated the mechanisms of potential emergence of resistance to ABT-737. MCL1 has been shown to confer resistance to ABT-737 because of the reduced affinity of ABT-737 for MCL1. Studies by Lestini, and co-workers have shown that in NB cells, resistance to ABT-737 can be overcome by MCL1 knockdown [51]. Currently, available data 
suggest the utility of effective target identification on tumor specimens to stratify responders and the formulation of drug combination regimens with MCL1 antagonists to enhance the clinical effectiveness of agents such as ABT-737 in future clinical trials [51, 52 ]. Compared to many NB cell lines, NB tumor specimens expressed high BCL2 [53]. The anti-tumor activity of ABT263 against cell lines with high BCL2 cell lines suggested the potential of targeting BCL2 for effective therapeutics [53].

Agents that target IAPs have also been evaluated in preclinical models of NB. Generally, two distinct approaches are being taken in the development and identification of effective inhibitors of IAP: antisense oligonucleotides and small molecular weight inhibitors [54]. Antisense oligonucleotides against XIAP and BIRC5 are already been evaluated in preclinical and early phase clinical trials for adult malignancies. YM155 (1-(2-Methoxyethyl)-2-methyl-4,9-dioxo-3(pyrazin-2-ylmethyl)-4,9-dihydro- $1 H$-naphtho[2,3- $d$ ]imidazolium bromide) has been shown to inhibit BIRC5 expression in a dose and time dependent manner leading to the activation of caspases in a variety of tumor models. Currently, YM155 has been evaluated in early phase clinical trials for adult tumors [55]. The effect of YM155 against a panel of NB cell lines have been examined, which showed that YM155 induced effective cytotoxicity in 14 of the 23 neuroblastoma cell lines, with an $\mathrm{IC}_{50}$ in the low $\mathrm{nM}$ range, although a direct correlation between the $\mathrm{IC}_{50}$ values in individual cell lines and extent of BIRC5 expression was not noted in this study [56]. However, mRNA array studies identified the expression of ABCB1 (MDR1) as the most predictive gene for the generation of resistance to YM155 and it was possible to sensitize resistant cells by ABCB1 knockdown.

Recently, a number of innovative screening approaches have been attempted to identify agents and drug combinations that target apoptotic pathways in NB. Tsang and colleagues have used a synthetic lethal screen approach to discover targets for effective therapeutic combinations with topotecan [57]. Their studies have found a number of genes whose suppression synergized with toptecan to enhance cell death. Notable among these were the NF- $\kappa B$ target genes. Furthermore, in drug combinations, known NF- $\kappa$ B inhibitors such as bortezomib were also found to induce caspase-3 activity in NB cell lines and delay tumor formation in xenograft mouse models. Specific molecular aberrations in NB and associated anti-apoptotic changes have also been used in drug screening studies. Recently, Zirath et al. have screened a library of 80 cytotoxic compounds to identify those that preferentially targeted the cells with MYC over-expression [58]. These studies have shown that MYC also increases sensitivity to targeted inhibition of certain cellular mechanisms including the activity of topoisomerases and the mitotic control machinery. In addition to cell lines, methods to screen for agents that selectively target patientderived stem-like or tumor-initiating cells (TICs) havealso been described [59]. The dequalinium analogue, C-14 linker (DECA-14), and rapamycin showed selective inhibition of NB TICs in vitro and a reduction in xenograft tumor growth and tumor initiating capacity.

\section{Discussion}

In comparison to the progress made in the treatment outcomes of a number of common pediatric malignancies, the survival rates of children diagnosed with NB with unfavorable 
biological features still remains unacceptably low. Hence, in the recent past a significant amount of research effort has been focused on the development of effective novel therapeutic approaches for the treatment of these children. With the application of cutting edge molecular technologies, recent years have seen a significant advancement in new knowledge regarding the complex molecular components and pathways involved in the diversity, growth, survival, differentiation, metastasis and treatment resistance of this disease. It is becoming evident that the over-expression of oncogenic survival factors and effective interference with normal cell death pathways appear to be key strategic characteristics of aggressive NB. As details of the components, role and regulators of the intrinsic apoptotic pathway in cancer emerge, it is expected that newer agents and novel therapeutic approaches, especially those with mechanistically validated drug combination regimens, will be developed for the treatment of refractory NB. In addition, the advent of molecular screening techniques such as Whole Genome Sequencing and Comparative Genomic Hybridization arrays may facilitate the screening of NB specimens from individual patients in high-throughput approach for target validation to advance future individualized therapeutic regimens.

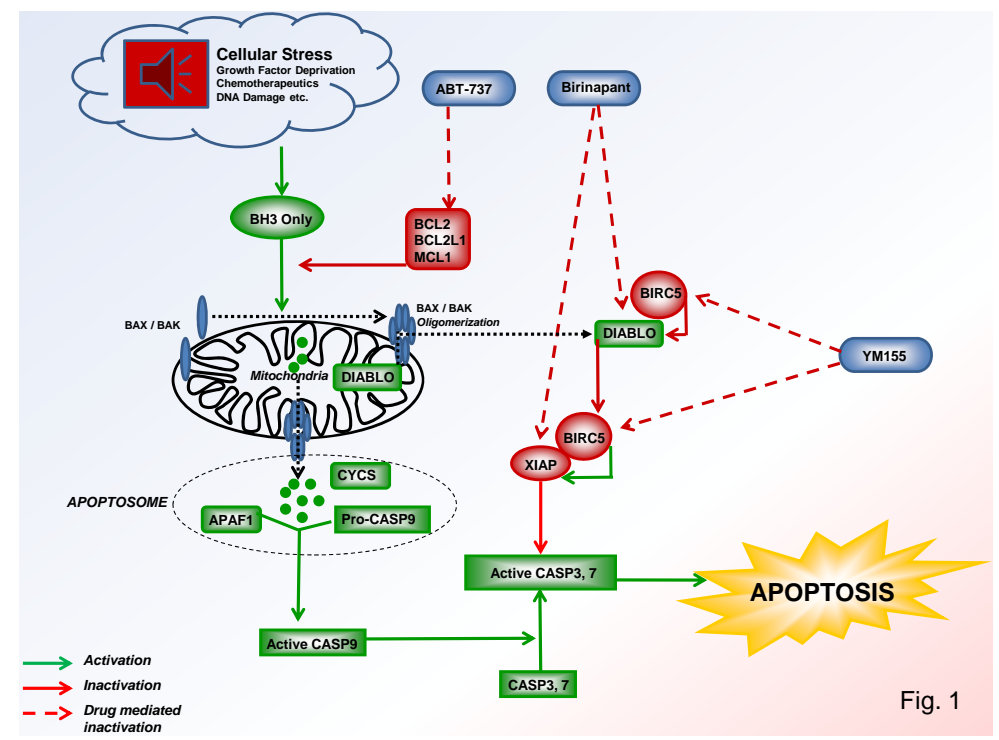

Figure 1. Schematic representation of key events of intrinsic apoptotic pathways.

The intrinsic pathway is triggered by stimuli from cytotoxic stress which leads to the upregulation of $\mathrm{BH} 3$ only proteins and consequently the mitochondrial translocation and oligomerization of BAX/BAK. This results in the release of cytochrome $\mathrm{c}$ which then binds to the pro-apoptotic factor Apaf-1 to form apoptosomes. Aptoptosomes then activate caspase-9, which in turn leads to the activation of caspases-3, 7 and subsequently to apoptosis. This process can be regulated by XIAP. In addition, the mitochondrial activation also leads to the release of SMAC /DIABLO which promotes apoptosis by directly interacting with IAPs and 
disrupting their ability to inactivate the caspases but itself can be modulated by BIRC5. In addition to IAPs, mitochondrial apoptosis can also be inhibited by the anti-apoptotic BCL2 family members such as BCL2, MCL1 and BCL- $\mathrm{X}_{\mathrm{L}}$. The points at which different targeted agents may interfere with their activities are also indicated.

\section{Acknowledgements}

We thank Prof. H.N. Caron, Prof. R. Versteegand and Dr. Molenaar for providing the research training that was invaluable in writing this review (FL). We acknowledge the technical assistance of Gaya Narendran in the preparation of Fig. 1.

This study was supported in part by a grant for AN from the Alberta Children's Hospital Foundation, Calgary, Alberta, Canada.

\section{Author details}

Fieke Lamers ${ }^{1}$ and Aru Narendran ${ }^{2}$

1 Department of Oncogenomics, Academic Medical Center, University of Amsterdam, AZ Amsterdam, The Netherlands

2 Laboratory for Preclinical and Drug Discovery Studies, Pediatric Oncology Experimental Therapeutics Investigators' Consortium (POETIC). Division of Pediatric Oncology, Alberta Children's Hospital, University of Calgary, Calgary, Alberta, Canada

\section{References}

[1] Goldsmith, K. C. Hogarty MD Targeting programmed cell death pathways with experimental therapeutics: opportunities in high risk neuroblastoma. Cancer Lett. (2005). Oct 18;228(1-2):133-41.

[2] Ouyang, L, Shi, Z, Zhao, S, Wang, F. T, Zhou, T. T, Liu, B, \& Bao, J. K. Programmed cell death pathways in cancer: a review of apoptosis, autophagy and programmed necrosis. Cell Prolif. (2012). Dec;, 45(6), 487-98.

[3] García-sáez, A. J. The secrets of the Bcl-2 family. Cell Death Differ. (2012). Nov; 19(11), 1733-40.

[4] Li, P, Nijhawan, D, Budihardjo, I, Srinivasula, S. M, Ahmad, M, Alnemri, E. S, \& Wang, X. Cytochrome $c$ and dATP-dependent formation of Apaf-1/caspase-9 complex initiates an apoptotic protease cascade. Cell. (1997). Nov 14; 91(4), 479-89. 
[5] Adams, J. M, \& Cory, S. Apoptosomes: engines for caspase activation. Curr Opin Cell Biol. (2002). Dec;, 14(6), 715-20.

[6] Huang, Z. Bcl-2 family proteins as targets for anticancer drug design. Oncogene. (2000). Dec 27; 19(56), 6627-31.

[7] Danial, N. N. BCL-2 family proteins: critical checkpoints of apoptotic cell death. Clin Cancer Res. (2007). Dec 15; 13(24), 7254-63.

[8] Wang, K, Yin, X. M, Chao, D. T, Milliman, C. L, \& Korsmeyer, S. J. BID: a novel BH3 domain-only death agonist. Genes Dev. (1996). Nov 15;, 10(22), 2859-69.

[9] Chao, D. T, \& Korsmeyer, S. J. BCL-2 family: regulators of cell death. Annu Rev Immunol. (1998). , 16, 395-419.

[10] Reed, J. C. Dysregulation of apoptosis in cancer. J Clin Oncol. (1999). Sep; 17(9), 2941-53.

[11] Coultas, L. Strasser A The role of the Bcl-2 protein family in cancer. Semin Cancer Biol. (2003). Apr; 13(2), 115-23.

[12] Fulda, S, \& Debatin, K. M. Caspase activation in cancer therapy, in: M. Los, H. Walczak (Eds.), Caspases-Their Role in Cell Death and Cell Survival, Kluwer Academic Press, (2002).

[13] Ikeda, H, Hirato, J, Akami, M, Matsuyama, S, Suzuki, N, Takahashi, A, \& Kuroiwa, M. Bcl-2 oncoprotein expression and apoptosis in neuroblastoma. J Pediatr Surg. (1995). Jun;, 30(6), 805-8.

[14] Hagenbuchner, J, Ausserlechner, M. J, Porto, V, David, R, Meister, B, Bodner, M, Villunger, A, Geiger, K, \& Obexer, P. The anti-apoptotic protein BCL2L1/Bcl-xL is neutralized by pro-apoptotic PMAIP1/Noxa in neuroblastoma, thereby determining bortezomib sensitivity independent of prosurvival MCL1 expression. J Biol Chem. (2010). Mar 5;, 285(10), 6904-12.

[15] Mohan, N, Banik, N. L, \& Ray, S. K. Synergistic efficacy of a novel combination therapy controls growth of Bcl-x(L) bountiful neuroblastoma cells by increasing differentiation and apoptosis. Cancer Biol Ther. (2011). Nov 1; 12(9), 846-54.

[16] Mohan, N, Banik, N. L, \& Ray, S. K. Synergistic efficacy of a novel combination therapy controls growth of $\mathrm{Bcl}-\mathrm{x}(\mathrm{L})$ bountiful neuroblastoma cells by increasing differentiation and apoptosis. Cancer Biol Ther. (2011). Nov 1; 12(9), 846-54.

[17] Tamm, I, Kornblau, S. M, Segall, H, Krajewski, S, Welsh, K, Kitada, S, Scudiero, D. A, Tudor, G, Qui, Y. H, Monks, A, Andreeff, M, \& Reed, J. C. Expression and prognostic significance of IAP-family genes in human cancers and myeloid leukemias. Clin Cancer Res. (2000). May;, 6(5), 1796-803.

[18] Cossu, F, Milani, M, Vachette, P, Malvezzi, F, Grassi, S, Lecis, D, Delia, D, Drago, C, Seneci, P, Bolognesi, M, \& Mastrangelo, E. Structural insight into inhibitor of apopto- 
sis proteins recognition by a potent divalent smac-mimetic. PLoS One. (2012). e49527. doi:journal.pone.0049527.

[19] De Almagro, M. C, \& Vucic, D. The inhibitor of apoptosis (IAP) proteins are critical regulators of signaling pathways and targets for anti-cancer therapy. Exp Oncol. (2012). Oct; 34(3), 200-11.

[20] Vucic, D, Dixit, V. M, \& Wertz, I. E. Ubiquitylation in apoptosis: a post-translational modification at the edge of life and death. Nat Rev Mol Cell Biol. (2011). Jun 23; 12(7), 439-52.

[21] Vaux, D. L, \& Silke, J. Mammalian mitochondrial IAP binding proteins. Biochem Biophys Res Commun. (2003). May 9;, 304(3), 499-504.

[22] Song, Z, Yao, X, \& Wu, M. Direct interaction between survivin and Smac/DIABLO is essential for the anti-apoptotic activity of survivin during taxol-induced apoptosis. J Biol Chem. (2003). Jun 20;, 278(25), 23130-40.

[23] Dohi, T, Okada, K, Xia, F, Wilford, C. E, Samuel, T, Welsh, K, Marusawa, H, Zou, H, Armstrong, R, Matsuzawa, S, Salvesen, G. S, Reed, J. C, \& Altieri, D. C. An IAP-IAP complex inhibits apoptosis. J Biol Chem. (2004). Aug 13;, 279(33), 34087-90.

[24] Kelly, R. J, Lopez-chavez, A, Citrin, D, Janik, J. E, \& Morris, J. C. Impacting tumor cell-fate by targeting the inhibitor of apoptosis protein survivin. Mol Cancer. (2011). Apr 6;10:35.

[25] Islam, A, Kageyama, H, Takada, N, Kawamoto, T, Takayasu, H, Isogai, E, Ohira, M, Hashizume, K, Kobayashi, H, Kaneko, Y, \& Nakagawara, A. High expression of Survivin, mapped to $17 q 25$, is significantly associated with poor prognostic factors and promotes cell survival in human neuroblastoma. Oncogene. (2000). Feb 3;, 19(5), 617-23.

[26] Hoffman, W. H, Biade, S, Zilfou, J. T, Chen, J, \& Murphy, M. Transcriptional repression of the anti-apoptotic survivin gene by wild type J Biol Chem. (2002). Feb 1;277(5):3247-57., 53.

[27] Hossain, M. M, Banik, N. L, \& Ray, S. K. Survivin knockdown increased anti-cancer effects of (-)-epigallocatechin-3-gallate in human malignant neuroblastoma SK-N-BE2 and SH-SY5Y cells. Exp Cell Res. (2012). Aug 1;, 318(13), 1597-610.

[28] Zangemeister-wittke, U, \& Simon, H. U. An IAP in action: the multiple roles of survivin in differentiation, immunity and malignancy. Cell Cycle. (2004). Sep; 3(9), 1121-3.

[29] Tamm, I, Wang, Y, Sausville, E, Scudiero, D. A, Vigna, N, Oltersdorf, T, \& Reed, J. C. IAP-family protein survivin inhibits caspase activity and apoptosis induced by Fas (CD95), Bax, caspases, and anticancer drugs. Cancer Res. (1998). Dec 1; 58(23), 5315-20. 
[30] Chandele, A, Prasad, V, Jagtap, J. C, Shukla, R, \& Shastry, P. R. Upregulation of survivin in G2/M cells and inhibition of caspase 9 activity enhances resistance in staurosporine-induced apoptosis. Neoplasia. (2004). Jan-Feb;, 6(1), 29-40.

[31] Lamers, F, Van Der Ploeg, I, Schild, L, Ebus, M. E, Koster, J, Hansen, B. R, Koch, T, Versteeg, R, Caron, H. N, \& Molenaar, J. J. Knockdown of survivin (BIRC5) causes apoptosis in neuroblastoma via mitotic catastrophe. Endocr Relat Cancer. (2011). Oct $27 ; 18(6), 657-68$.

[32] Lens, S. M, Vader, G, \& Medema, R. H. The case for Survivin as mitotic regulator. Curr Opin Cell Biol. (2006). Dec;, 18(6), 616-22.

[33] Li, F, Yang, J, Ramnath, N, Javle, M. M, \& Tan, D. Nuclear or cytoplasmic expression of survivin: what is the significance? Int J Cancer. (2005). Apr 20; 114(4), 509-12.

[34] Miller, M. A, Ohashi, K, Zhu, X, Mcgrady, P, London, W. B, Hogarty, M, \& Sandler, A. D. Survivin mRNA levels are associated with biology of disease and patient survival in neuroblastoma: a report from the children's oncology group. J Pediatr Hematol Oncol. (2006). Jul;, 28(7), 412-7.

[35] Teitz, T, Lahti, J. M, \& Kidd, V. J. Aggressive childhood neuroblastomas do not express caspase-8: an important component of programmed cell death. J Mol Med (Berl). (2001). Aug;, 79(8), 428-36.

[36] Eggert, A, Grotzer, M. A, Zuzak, T. J, Wiewrodt, B. R, Ho, R, Ikegaki, N, \& Brodeur, G. M. Resistance to tumor necrosis factor-related apoptosis-inducing ligand (TRAIL)induced apoptosis in neuroblastoma cells correlates with a loss of caspase-8 expression. Cancer Res. (2001). Feb 15; 61(4), 1314-9.

[37] Van Noesel, M. M, Van Bezouw, S, Voûte, P. A, Herman, J. G, Pieters, R, \& Versteeg, R. Clustering of hypermethylated genes in neuroblastoma. Genes Chromosomes Cancer. (2003). Nov;, 38(3), 226-33.

[38] Kasof, G. M, \& Gomes, B. C. Livin, a novel inhibitor of apoptosis protein family member. J Biol Chem. (2001). Feb 2;, 276(5), 3238-46.

[39] Kim, D. K, Alvarado, C. S, Abramowsky, C. R, Gu, L, Zhou, M, Soe, M. M, Sullivan, K, George, B, Schemankewitz, E, \& Findley, H. W. Expression of inhibitor-of-apoptosis protein (IAP) livin by neuroblastoma cells: correlation with prognostic factors and outcome. Pediatr Dev Pathol. (2005). Nov-Dec;, 8(6), 621-9.

[40] Dasgupta, A, Peirce, S. K, \& Findley, H. W. MycN is a transcriptional regulator of livin in neuroblastoma. Oncol Rep. (2009). Oct;, 22(4), 831-5.

[41] Fulda, S, Lutz, W, Schwab, M, \& Debatin, K. M. MycN sensitizes neuroblastoma cells for drug-induced apoptosis. Oncogene. (1999). Feb 18;, 18(7), 1479-86. 
[42] Dasgupta, A, Alvarado, C. S, Xu, Z, \& Findley, H. W. Expression and functional role of inhibitor-of-apoptosis protein livin (BIRC7) in neuroblastoma. Biochem Biophys Res Commun. (2010). Sep 10;, 400(1), 53-9.

[43] Chen, Z, Naito, M, Hori, S, Mashima, T, Yamori, T, \& Tsuruo, T. A human IAP-family gene, apollon, expressed in human brain cancer cells. Biochem Biophys Res Commun. (1999). Nov 2; 264(3), 847-54.

[44] Hao, Y, Sekine, K, Kawabata, A, Nakamura, H, Ishioka, T, Ohata, H, Katayama, R, Hashimoto, C, Zhang, X, Noda, T, Tsuruo, T, \& Naito, M. Apollon ubiquitinates SMAC and caspase-9, and has an essential cytoprotection function. Nat Cell Biol. (2004). Sep; 6(9), 849-60.

[45] Qiu, X. B, \& Goldberg, A. L. The membrane-associated inhibitor of apoptosis protein, BRUCE/Apollon, antagonizes both the precursor and mature forms of Smac and caspase-9. J Biol Chem. (2005). Jan 7;, 280(1), 174-82.

[46] Ismail, E. A, Mahmoud, H. M, Tawfik, L. M, Habashy, D. M, Adly, A. A, Sherif, N. H, \& Abdelwahab, M. A. BIRC6/Apollon gene expression in childhood acute leukemia: impact on therapeutic response and prognosis. Eur J Haematol. (2012). Feb;, 88(2), 118-27.

[47] Lamers, F, Schild, L, Koster, J, Speleman, F, Ora, I, Westerhout, E. M, Van Sluis, P, Versteeg, R, Caron, H. N, \& Molenaar, J. J. Identification of BIRC6 as a novel intervention target for neuroblastoma therapy. BMC Cancer. (2012). Jul 12;12:285.

[48] Stamelos, V. A, Redman, C. W, \& Richardson, A. Understanding sensitivity to BH3 mimetics: ABT-737 as a case study to foresee the complexities of personalized medicine. J Mol Signal. (2012). Aug 16;7(1):12. doi:

[49] Klymenko, T, Brandenburg, M, Morrow, C, Dive, C, \& Makin, G. The novel Bcl-2 inhibitor ABT-737 is more effective in hypoxia and is able to reverse hypoxia-induced drug resistance in neuroblastoma cells. Mol Cancer Ther. (2011). Dec;, 10(12), 2373-83.

[50] Goldsmith, K. C, Lestini, B. J, Gross, M, Ip, L, Bhumbla, A, Zhang, X, Zhao, H, Liu, X, \& Hogarty, M. D. BH3 response profiles from neuroblastoma mitochondria predict activity of small molecule Bcl-2 family antagonists. Cell Death Differ. (2010). May;" 17(5), 872-82.

[51] Lestini, B. J, Goldsmith, K. C, Fluchel, M. N, Liu, X, Chen, N. L, Goyal, B, Pawel, B. R, \& Hogarty, M. D. Mcl1 downregulation sensitizes neuroblastoma to cytotoxic chemotherapy and small molecule Bcl2-family antagonists. Cancer Biol Ther. (2009). Aug;, 8(16), 1587-95.

[52] Goldsmith, K. C, Gross, M, Peirce, S, Luyindula, D, Liu, X, Vu, A, Sliozberg, M, Guo, R, Zhao, H, Reynolds, C. P, \& Hogarty, M. D. Mitochondrial Bcl-2 family dynamics 
define therapy response and resistance in neuroblastoma. Cancer Res. (2012). May $15 ;$; $72(10), 2565-77$.

[53] Lamers, F, Schild, L, Koster, J, Versteeg, R, Caron, H. N, \& Molenaar, J. J. Targeted BIRC5 silencing using YM155 causes cell death in neuroblastoma cells with low ABCB1 expression. Eur J Cancer. (2012). Mar;, 48(5), 763-71.

[54] Schimmer, A. D, \& Dalili, S. Targeting the IAP family of caspase inhibitors as an emerging therapeutic strategy. Hematology Am Soc Hematol Educ Program. (2005). , 2005, 215-9.

[55] Tolcher, A. W, Mita, A, Lewis, L. D, Garrett, C. R, Till, E, Daud, A. I, Patnaik, A, Papadopoulos, K, Takimoto, C, Bartels, P, Keating, A, \& Antonia, S. Phase I and pharmacokinetic study of YM155, a small-molecule inhibitor of survivin. J Clin Oncol. (2008). Nov 10;, 26(32), 5198-203.

[56] Lamers, F, Schild, L, Koster, J, Versteeg, R, Caron, H. N, \& Molenaar, J. J. Targeted BIRC5 silencing using YM155 causes cell death in neuroblastoma cells with low ABCB1 expression. Eur J Cancer. (2012). Mar;, 48(5), 763-71.

[57] Tsang, P. S, Cheuk, A. T, Chen, Q. R, Song, Y. K, Badgett, T. C, Wei, J. S, \& Khan, J. Synthetic lethal screen identifies NF- $\kappa \mathrm{B}$ as a target for combination therapy with topotecan for patients with neuroblastoma. BMC Cancer. (2012). Mar 21;12:101

[58] Frenzel, A, Zirath, H, Vita, M, Albihn, A, \& Henriksson, M. A. Identification of cytotoxic drugs that selectively target tumor cells with MYC overexpression. PLoS One. (2011). e27988. doi:journal.pone.0027988. Epub 2011 Nov 23.

[59] Smith, K. M, Datti, A, Fujitani, M, Grinshtein, N, Zhang, L, Morozova, O, Blakely, K. M, Rotenberg, S. A, Hansford, L. M, Miller, F. D, Yeger, H, Irwin, M. S, Moffat, J, Marra, M. A, Baruchel, S, Wrana, J. L, \& Kaplan, D. R. Selective targeting of neuroblastoma tumour-initiating cells by compounds identified in stem cell-based small molecule screens. EMBO Mol Med. (2010). Sep; 2(9), 371-84. 
